\title{
HIGHLIGHTS
}

$\Rightarrow$ NEURODEGENERATIVE DISEASE

\section{A route for pathology?}

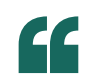

inoculated

16-month-

old wild-type

mice exhibited

markers

of $\alpha$-Syn

pathology in

the brainstem

and a decrease

in dopamine

levels in the

striatum

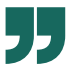

Parkinson disease (PD) is associated with the development of aggregates of the protein $\alpha$-synuclein ( $\alpha$-Syn) in the CNS and various peripheral tissues, including those of the gastrointestinal (GI) tract. GI dysfunction is a common non-motor symptom of PD and frequently precedes the emergence of the motor symptoms typically associated with the disease. One hypothesis states that pathological forms of $\alpha$-Syn are transmitted via vagal nerve fibres - which innervate the enteric nervous system (ENS) - to the brain, where they eventually cause motor dysfunction. However, it is not clear how pathological $\alpha$-Syn affects or may spread in the ENS. Here, Challis, Hori et al. show in mice that GI inoculation of $\alpha$-Syn fibrils can induce physiological changes in the ENS and GI dysfunction. Moreover, in aged mice, such treatment can also result in the development of $\alpha$-Syn pathology in the brain and motor deficits.

The authors first examined whether pathological $\alpha$-Syn can cause GI dysfunction. To do so, they delivered $\alpha$-Syn preformed fibrils

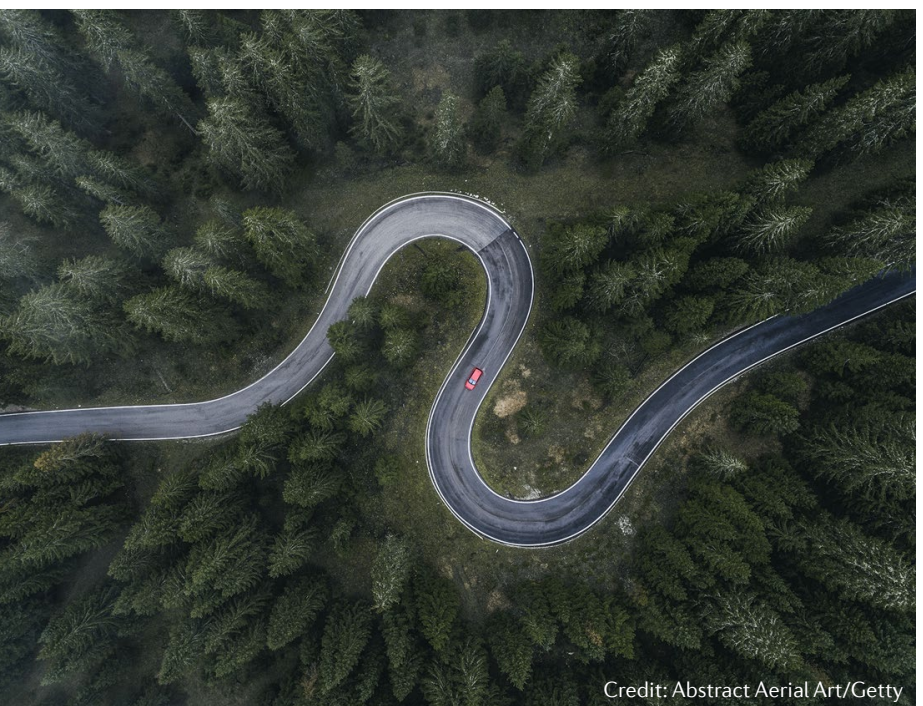

(PFFs) - often used to model $\alpha$-Syn pathology - into the duodenum of young adult (8-10-week-old) wild-type mice; this part of GI tract was selected because it is innervated by vagal fibres. Sixty days after $\alpha$-Syn PFF inoculation, the animals began to show signs of GI dysfunction.

The authors next examined the initial effects of the $a$-Syn PFFs on the duodenal tissue. A week after the inoculation, interleukin-6 (IL-6) levels rose. This cytokine can promote the survival of enteric neurons, so the authors investigated how the $\alpha$-Syn PFFs affected the ENS. They found that alongside the rise in IL-6 levels, enteric glial cells also increased in number, indicating that $\alpha$-Syn PFFs induce reactive gliosis. The activation of such glia, and enteric neurons, can lead to the release of cytokines that can recruit macrophages. In line with this, the authors detected increases in two cytokines known to recruit macrophages to the gut and in a marker of these cells. Thus, these data indicate that $\alpha$-Syn PFFs initiate an inflammatory response that aims to protect the ENS.

Over the first 60 days following the inoculation, there were rises in markers for pathological $\alpha$-Syn, which then declined by 120 days. There was also an initial decline after inoculation in the level of glucocerebrosidase (GCase), a lysosomal enzyme that may be inhibited by pathological $\alpha$-Syn, but this enzyme recovered to normal levels by the end of the 120-day period. Together, these data suggest that, in response to $\alpha$-Syn PFF inoculation, protein homeostatic mechanisms were initiated to counter the introduced $\alpha$-Syn pathology.

The authors next further examined the relationship between GCase and pathological $\alpha-S y n$.
To do so, they used the a-Synoverexpressing (ASO) mouse model of synucleinopathy. The authors confirmed that these mice show marked deficits in GI function and found that these animals exhibit a decrease in duodenal GCase levels. Virus-mediated re-expression of GCase in the duodenum reduced a-Syn pathology locally and ameliorated the GI dysfunction in these mice.

As a-Syn fibrils can impair neurotransmission, the authors investigated whether pathological a-Syn affects ENS connectivity. The authors used an optogenetic approach with calcium imaging to examine activity in the ENS. Following $a$-Syn PFF inoculation in wild-type mice, the responses of the duodenal neurons decreased in response to photostimulation, suggesting that the ENS network was exhibiting abnormal connectivity. Enteric neurons from ASO mice also showed similar deficits in connectivity, which could be partially rescued through GCase expression.

In the final part of the study, the authors examined whether the inoculation of the duodenum with a-Syn PFFs could affect the brain. The young adult mice showed no marked $\alpha$-Syn pathology in the brain or behavioural impairments after 120 days. By contrast, after the same period, inoculated 16-month-old wild-type mice exhibited markers of $\alpha$-Syn pathology in the brainstem and a decrease in dopamine levels in the striatum, a region implicated in PD. Moreover, these mice showed a decline in GI function and sensorimotor deficits.

Together, these findings show in mice that $\alpha$-Syn fibrils delivered into the gut can cause GI dysfunction and, in aged animals, effects in the brain.

Darran Yates

ORIGINAL ARTICLE Challis, C. et al. Gut-seeded $\alpha$-synuclein fibrils promote gut dysfunction and $\alpha$-synuclein fibrils promote gut dysfunction and
brain pathology specifically in aged mice. Nat. Neurosci. https://doi.org/10.1038/s41593-0200589-7 (2020) 